\title{
On solving periodic differential matrix equations with applications to periodic system norms computation
}

\author{
A. Varga
}

\begin{abstract}
Periodic Lyapunov, Sylvester and Riccati differential equations have many important applications in the analysis and design of linear periodic control systems. For the numerical solution of these equations efficient numerically reliable algorithms based on the periodic Schur decomposition are proposed. The new multi-shot type algorithms compute periodic solutions in an arbitrary number of time moments within one period by employing suitable discretizations of the continuous-time problems. In contrast to traditionally used oneshot periodic generator methods, the multi-shot type methods have the advantage to be able to address problems with large periods and/or unstable dynamics. Applications of the proposed techniques to compute several system norms are presented.
\end{abstract}

\section{INTRODUCTION}

We consider the numerical computation of the periodic solutions to three classes of periodic matrix differential equations:

1) Periodic Lyapunov differential equations (PLDE) either in the direct form

$$
\dot{X}(t)=A(t) X(t)+X(t) A^{T}(t)+Q(t)
$$

or in the adjoint form

$$
-\dot{X}(t)=A^{T}(t) X(t)+X(t) A(t)+\widetilde{Q}(t)
$$

where $Q(t)=Q^{T}(t), \widetilde{Q}(t)=\widetilde{Q}^{T}(t)$ and $A(t), Q(t)$, and $\widetilde{Q}(t)$ are $n \times n T$-periodic matrices (i.e., $\forall t A(t+T)=A(t)$, $Q(t+T)=Q(t), \widetilde{Q}(t+T)=\widetilde{Q}(t))$. These equations play an important role in the analysis of controllability/observability of linear continuous-time periodic systems [1], in solving periodic stabilization problems [2], computing Hankel- and $\mathcal{H}_{2}$-norms of periodic systems [3], or in solving periodic differential Riccati equations by employing Newtons' method [4].

2) Periodic Sylvester differential equation (PSDE) of the form

$$
\dot{X}(t)=A(t) X(t)+X(t) B(t)+C(t)
$$

where $A(t), B(t)$, and $C(t)$ are respectively $m \times m, n \times n$, and $m \times n T$-periodic matrices. The PDSE (3) is useful in solving periodic eigenvalue assignment problems [5], [6].

3) Periodic Riccati differential equation (PRDE) in the filtering form

$$
\dot{X}(t)=A(t) X(t)+X(t) A^{T}(t)+R(t)-X(t) Q(t) X(t)
$$

This work was partially supported via the Swedish Strategic Research Foundation Grant "Matrix Pencil Computations in Computer-Aided Control System Design: Theory, Algorithms and Software Tools".

A. Varga is with the German Aerospace Center, DLR - Oberpfaffenhofen, Institute of Robotics and Mechatronics, D-82234 Wessling, Germany; Email: andras.vargaedlr.de or in the control form

$$
-\dot{X}(t)=A^{T}(t) X(t)+X(t) A(t)+Q(t)-X(t) R(t) X(t)
$$

where $Q(t)=Q^{T}(t) \geq 0, R(t)=R^{T}(t) \geq 0$, and $A(t)$, $Q(t), R(t)$ are $n \times n T$-periodic matrices. Solving these equations for the stabilizing solution plays an important role in periodic filtering or control problems [7], [4], [3].

In this paper we propose reliable numerical methods to compute the periodic solution $X(t)=X(t+T)$ of equations (1)-(5). Both one-shot periodic generator methods as well as multi-shot type methods are discussed. The multi-shot type methods rely on discretization techniques, which turn the continuous-time problems into equivalent discrete-time problems for which satisfactory numerical methods already exist. The main appeal of these methods is that the periodic solution $X(t)$ is computed simultaneously in many time moments within one period, so that the numerical difficulties related to numerical integrations for large periods and/or unstable dynamics are highly alleviated. The solution for intermediary values of time $t$ can be computed using special numerical integration by initializing the solution in the nearest time moment. Applications of the proposed techniques to compute the Hankel-, $\mathcal{H}_{2}$ - and $\mathcal{H}_{\infty}$-norms of a periodic system are presented.

The key numerical ingredient for solving the discretetime problems is the periodic real Schur form (PRSF) of a periodic matrix sequence [8], [9]. The underlying computational methods to solve discrete-time periodic Lyapunov [10] and Sylvester equations [11] employ the PRSF to reduce the problem matrices to condensed forms which allows an easy solution of the reduced equations. In the case of the solution of PRDEs, the main technique is the computation of an ordered PRSF of a symplectic periodic matrix together with the corresponding periodic basis of the stable invariant subspace [8], [9].

\section{SOLUTION OF PLDES}

Let $\Phi_{A}(t, \tau)$ denote the transition matrix corresponding to $A(t)$ satisfying

$$
\frac{\partial \Phi_{A}(t, \tau)}{\partial t}=A(t) \Phi_{A}(t, \tau), \quad \Phi_{A}(\tau, \tau)=I
$$

For a $T$-periodic $A(t), \Psi_{A}(\tau):=\Phi_{A}(\tau+T, \tau)$ is called the monodromy matrix corresponding to $A(t)$ and its eigenvalues, which are independent of $\tau$, are called characteristic multipliers. We say that $A(t)$ is a stable periodic matrix if all its characteristic multipliers have magnitudes less than 1. 
Existence conditions of periodic solutions of PLDEs have been discussed in [12]. We recall the main result of [12].

Theorem 1: The PLDE (1) or (2) admits a unique $T$ periodic solution $X(t)$ if and only if $\Psi_{A}(\tau)$ does not have reciprocal eigenvalues.

In what follows, we assume the above condition fulfilled.

\section{A. Periodic generator method}

The computation of the solution of (1) can be done in two steps using the periodic generator method. First we solve for a given time, say $t=0$, the standard discrete-time Lyapunov equation satisfied by $X(0)(=X(T))$ [1]

$$
X(0)=\Psi_{A}(0) X(0) \Psi_{A}^{T}(0)+W(T, 0)
$$

where

$$
W\left(t, t_{0}\right):=\int_{t_{0}}^{t} \Phi_{A}(t, \tau) Q(\tau) \Phi_{A}^{T}(t, \tau) d \tau
$$

Then, we integrate from $t=0$ to $t=T$ the matrix differential equation (1) using any standard integration method for ODEs. Observe that because of the symmetry of $X(t)$, only $\frac{n(n+1)}{2}$ equations must be integrated.

For the adjoint Lyapunov differential equation (2) a similar approach can be used with obvious replacements. First we solve the standard discrete-time Lyapunov equation satisfied by $X(T)(=X(0))$

$$
X(T)=\Psi_{A}^{T}(0) X(T) \Psi_{A}(0)+\widetilde{W}(T, 0)
$$

where

$$
\widetilde{W}\left(t_{f}, t\right):=\int_{t}^{t_{f}} \Phi_{A}^{T}(\tau, t) \widetilde{Q}(\tau) \Phi_{A}(\tau, t) d \tau
$$

and then integrate (2) backward in time from $t=T$ to $t=0$.

The one-shot approach has several potential numerical difficulties for long periods and/or unstable dynamics associated with $A(t)$. For example, the computation of the free term $W(T, 0)$ in (7) using the methods described in the next subsection can be hardly done with high accuracy for long periods. The same is true for evaluating the monodromy matrix $\Phi_{A}(T, 0)$ for large values of $T$. Moreover, for unstable $A(t)$ (i.e., some eigenvalues of $\Phi_{A}(T, 0)$ have magnitudes larger than 1), the differential equations are illconditioned and thus any numerical integration algorithm for ODEs becomes numerically unstable. This is why, generally the one-shot method is not recommended to solve periodic differential Lyapunov equations.

\section{B. Multi-shot approach}

The values of the solution $X(t)$ of the PDLE (1) at time moments $t$ and $t+\Delta$ are related as [1]

$X(t+\Delta)=\Phi_{A}(t+\Delta, t) X(t) \Phi_{A}^{T}(t+\Delta, t)+W(t+\Delta, t)$

Let $N \geq 1$ be an integer such that $\Delta:=T / N$ represents a meaningful time increment to determine the solution $X(t)$. Then, the solution at successive time moments $(k-1) \Delta$ and $k \Delta$ satisfies

$$
X_{k+1}=F_{k} X_{k} F_{k}^{T}+W_{k}
$$

where $X_{k}:=X((k-1) \Delta), F_{k}:=\Phi_{A}(k \Delta,(k-1) \Delta)$, and $W_{k}:=W(k \Delta,(k-1) \Delta)$. By imposing $X_{N+1}=X_{1}$, the $N$ coupled equations in (11) for $k=1, \ldots, N$ represent a discrete-time forward periodic Lyapunov equation. Thus, by solving the $N$ simultaneous equations (11), we determine $N$ values of the solution $X(t)$ at equidistant time instants. Since the time increment $\Delta$ can be chosen arbitrary small, this multi-shot approach certainly alleviates the numerical difficulties associated with large periods and/or unstable dynamics when evaluating $F_{k}$ and $W_{k}, k=1, \ldots, N$.

A similar approach can be developed to solve the adjoint PDLE (2). The solution $X(t)$ at time moments $t$ and $t-\Delta$ are related as [1]

$X(t-\Delta)=\Phi_{A}^{T}(t, t-\Delta) X(t) \Phi_{A}(t, t-\Delta)+\widetilde{W}(t, t-\Delta)$

Thus, the solution at successive time moments $(k-1) \Delta$ and $k \Delta$ satisfies

$$
X_{k}=F_{k}^{T} X_{k+1} F_{k}+\widetilde{W}_{k}
$$

where $\widetilde{W}_{k}:=\widetilde{W}(k \Delta,(k-1) \Delta)$. By imposing $X_{N+1}=$ $X_{1}$, the $N$ coupled equations in (12) for $k=1, \ldots, N$ represent a discrete-time backward periodic Lyapunov equation.

To solve the periodic discrete-time Lyapunov equations (11) and (12) the numerically reliable methods proposed in [10] can be used. These methods are based on computing the real Schur form (RSF) of the monodromy matrix $\Psi_{A}(0)=$ $F_{N} \cdots F_{2} F_{1}$ via the PRSF of the periodic matrix $F_{k}$. For the computation of PRSF numerically stable algorithms have been proposed in [8], [9] and robust numerical software implementations are available in the recently developed Periodic Systems Toolbox for Matlab [13]. For the solution of the periodic Lyapunov equations (11) and (12) robust numerical software is also available in this toolbox.

The computation of $F_{k}, W_{k}$ and $\widetilde{W}_{k}$ for $k=1, \ldots, N$ in the equations (11) and (12) can be done using numerical integration of appropriate ordinary differential equations (ODEs). To compute $F_{k}$, the matrix differential equation (6) must be integrated from $\tau=(k-1) \Delta$ to $k \Delta$ using appropriate methods for ODEs. Since the time step $\Delta$ can be chosen arbitrarily small, the numerical integration even for unstable $A(t)$ will not raise any numerical difficulties.

To compute $W_{k}$ observe that for given $t_{0}, Y(t):=$ $W\left(t, t_{0}\right)$ in (8) satisfies the Lyapunov differential equation

$$
\dot{Y}(t)=A(t) Y(t)+Y(t) A^{T}(t)+Q(t), Y\left(t_{0}\right)=0
$$

Thus, $W_{k}$ can be computed as $W_{k}=Y(k \Delta)$ by integrating the above equation from $t_{0}=(k-1) \Delta$ to $t_{f}=k \Delta$.

To compute $\widetilde{W}_{k}$ observe similarly that for given $t_{f}$, $\widetilde{Y}(t):=\widetilde{W}\left(t_{f}, t\right)$ in (10) satisfies the Lyapunov differential equation

$$
-\dot{\tilde{Y}}(t)=A(t) \tilde{Y}(t)+\tilde{Y}(t) A^{T}(t)+\widetilde{Q}(t), \tilde{Y}\left(t_{f}\right)=0
$$

Thus, $\widetilde{W}_{k}$ can be computed as $\widetilde{W}_{k}=\tilde{Y}((k-1) \Delta)$ by integrating the above equation backward in the time from $t_{f}=k \Delta$ to $t_{0}=(k-1) \Delta$.

To integrate the PLDEs (13) and (14) it is important to use methods which preserve the symmetry of the solution 
and if appropriate, also its positive definiteness. For example, methods which are able to preserve positive definiteness have been proposed in [14].

\section{SOLUTION OF PSDES}

Existence conditions similar to those for periodic solutions of PLDEs can be easily stated. The following result is a straightforward generalization of Theorem 1 and we give it without proof.

Theorem 2: The PSDE (3) admits a unique $T$-periodic solution $X(t)$ if and only if $\Psi_{A}(\tau)$ and $\Psi_{B}(\tau)$ do not have mutually reciprocal eigenvalues.

In this section, we assume the above condition is fulfilled.

\section{A. Periodic generator method}

The computation of the solution of the PSDE (3) can be done similarly to solving the PLDE (1). First we solve for a given time, say $t=0$, the standard discrete-time Sylvester equation satisfied by $X(0)(=X(T))$ [1]

$$
\begin{aligned}
X(0)= & \Phi_{A}(T, 0) X(0) \Phi_{B^{T}}^{T}(T, 0)+ \\
& \int_{0}^{T} \Phi_{A}(T, \tau) C(\tau) \Phi_{B^{T}}^{T}(T, \tau) d \tau
\end{aligned}
$$

Then, we integrate from $t=0$ to $t=T$ the matrix differential equation (3) using any standard integration method for ODEs. This one-shot approach leads to the same numerical difficulties as for PLDEs for long periods and/or unstable dynamics associated with $A(t)$ or $B(t)$. Thus, generally the one-shot methods are not recommended to solve periodic differential Sylvester equations.

\section{B. Multi-shot methods}

The values of the solution $X(t)$ of the PSDE (3) at time moments $t$ and $t+\Delta$ are related as [1]

$$
\begin{aligned}
X(t+\Delta)= & \Phi_{A}(t+\Delta, t) X(t) \Phi_{B^{T}}^{T}(t+\Delta, t)+ \\
& \int_{t}^{t+\Delta} \Phi_{A}(t+\Delta, \tau) C(\tau) \Phi_{B^{T}}^{T}(t+\Delta, \tau) d \tau
\end{aligned}
$$

Let $N \geq 1$ be an integer such that $\Delta:=T / N$ represents a meaningful time increment for the solution $X(t)$. Then, the solution at successive time moments $(k-1) \Delta$ and $k \Delta$ satisfies

$$
X_{k+1}=F_{k} X_{k} G_{k}^{T}+W_{k}
$$

where $X_{k}:=X((k-1) \Delta), F_{k}:=\Phi_{A}(k \Delta,(k-1) \Delta)$, $G_{k}:=\Phi_{B^{T}}(k \Delta,(k-1) \Delta)$ and

$$
W_{k}:=\int_{(k-1) \Delta}^{k \Delta} \Phi_{A}(k \Delta, \tau) C(\tau) \Phi_{B^{T}}^{T}(k \Delta, \tau) d \tau
$$

By imposing $X_{N+1}=X_{1}$, the $N$ coupled equations in (11) for $k=1, \ldots, N$ represent a discrete-time forward periodic Sylvester equation. To solve this equation the numerically reliable method proposed in [11] can be used. This method is based on the PRSFs of the periodic matrices $F_{k}$ and $G_{k}$.

The computation of $F_{k}, G_{k}$, and $W_{k}$ for $k=1, \ldots, N$ in the equation (15) can be done as follows. To compute $F_{k}$, the matrix differential equation (6) must be integrated from $\tau=(k-1) \Delta$ to $k \Delta$ using appropriate methods for ODEs. $G_{k}$ can be determined completely analogously with the obvious replacement of $A(t)$ by $B^{T}(t)$. To compute $W_{k}$ consider

$$
W(t):=\int_{t_{0}}^{t} \Phi_{A}(t, \tau) C(\tau) \Phi_{B^{T}}^{T}(t, \tau) d \tau
$$

which satisfies the Sylvester differential equation

$$
\dot{W}(t)=A(t) W(t)+W(t) B(t)+C(t), W\left(t_{0}\right)=0
$$

Thus, $W_{k}$ can be computed as $W(k \Delta)$ by integrating the above equation between $t_{0}=(k-1) \Delta$ and $t=k \Delta$.

\section{SOlution OF PRDES}

We address the computation of the periodic stabilizing solution $X(t)$ of the PRDE (4) or (5) for which $A(t)-$ $X(t) R(t)$ or, respectively, $A(t)-Q(t) X(t)$ is stable. The following result from [7] gives necessary and sufficient conditions for the existence of a stabilizing solution:

Theorem 3: The PRDE (4) or (5) admits a unique $T$ periodic stabilizing solution $X(t)=X^{T}(t) \geq 0$ if and only if the pair $(A(t), R(t))$ is stabilizable and the pair $(A(t), Q(t))$ is detectable.

In this section, we assume the above conditions are fulfilled.

\section{A. Periodic generator method}

Let $H(t)$ be the Hamiltonian matrix corresponding to the filtering PRDE (4)

$$
H(t)=\left[\begin{array}{cc}
-A^{T}(t) & Q(t) \\
R(t) & A(t)
\end{array}\right]
$$

or to the control PRDE (5)

$$
H(t)=\left[\begin{array}{cc}
A(t) & -R(t) \\
-Q(t) & -A^{T}(t)
\end{array}\right]
$$

In both cases $J H(t)+H^{T}(t) J=O$, where $J=\left[\begin{array}{cc}0 & I \\ -I & 0\end{array}\right]$, and thus $H(t)$ is indeed a Hamiltonian matrix. The theorem above ensures that the monodromy matrix $\Psi_{H}(0)$ is dichotomic and this property is the key aspect of the following approach to solve either the PRDEs (4) or (5) (see [15]):

1. Compute the symplectic transition matrix $\Phi_{H}(T, 0)$ (i.e., $\left.\Phi_{H}^{T}(T, 0) J \Phi_{H}(T, 0)=J\right)$ which has eigenvalues symmetric with respect to the unit circle.

2. Compute orthogonal $Z$ to reduce $\Phi_{H}(T, 0)$ to an ordered RSF such that

$$
Z^{T} \Phi_{H}(T, 0) Z=\left[\begin{array}{cc}
\Theta_{11} & \Theta_{12} \\
O & \Theta_{22}
\end{array}\right]
$$

where $\Theta_{11}$ has $n$ eigenvalues inside the unit circle and $\Theta_{22}$ has $n$ eigenvalues outside the unit circle.

3. Partition $Z$ in $n \times n$ blocks

$$
Z=\left[\begin{array}{ll}
Z_{11} & Z_{12} \\
Z_{21} & Z_{22}
\end{array}\right]
$$

and integrate from $t=0$ to $t=T$ the matrix differential equation

$$
\dot{S}(t)=H(t) S(t), S(0)=\left[\begin{array}{c}
Z_{11} \\
Z_{21}
\end{array}\right]
$$


From the conformably partitioned solution

$$
S(t)=\left[\begin{array}{l}
S_{1}(t) \\
S_{2}(t)
\end{array}\right]
$$

compute $X(t)=S_{2}(t) S_{1}^{-1}(t)$.

This approach is potentially numerically unreliable because it involves the numerical integration of two ODEs with unstable dynamics: the first to compute $\Phi_{H}(T, 0)$ and the second to compute $S(t)$. Therefore, for large periods, this approach will almost certainly fail because of the uncontrolled accumulation of roundoff errors.

\section{B. Multi-shot methods}

To alleviate the numerical difficulties related to the periodic generator method, we propose an alternative approach which relies on determining the transition matrix $\Phi_{H}(T, 0)$ in a product form (recommended in [16])

$$
\Phi_{H}(T, 0)=\Phi_{H}(T, T-\Delta) \cdots \Phi_{H}(2 \Delta, \Delta) \Phi_{H}(\Delta, 0)
$$

where $\Delta=T / N$ for a suitably chosen integer period $N$. We denote $H_{k}:=\Phi_{H}(k \Delta,(k-1) \Delta)$ for $k=1,2, \ldots$, which is obviously an $N$-periodic matrix. Using the algorithm of [8], we can determine an orthogonal $N$-periodic matrix $Z_{k}$ to reduce $H_{k}$ to an ordered PRSF such that

$$
Z_{k+1} H_{k} Z_{k}=\left[\begin{array}{cc}
J_{k ; 11} & J_{k ; 12} \\
O & J_{k ; 22}
\end{array}\right],
$$

where $J_{N ; 11} \cdots J_{2 ; 11} J_{1 ; 11}$ has $n$ eigenvalues inside the unit circle and $J_{N ; 22} \cdots J_{2 ; 22} J_{1 ; 22}$ has $n$ eigenvalues outside the unit circle. Since $\Phi_{H}(T, 0)=H_{N} \cdots H_{2} H_{1}$, it follows that $Z_{1}^{T} \Phi_{H}(T, 0) Z_{1}$ is in the ordered RSF (16), where both $\Theta_{11}=J_{N ; 11} \cdots J_{2 ; 11} J_{1 ; 11}$ and $\Theta_{22}=J_{N ; 22} \cdots J_{2 ; 22} J_{1 ; 22}$ are in RSF, and $Z$ is simply $Z_{1}$. If we partition $Z_{k}$ in $n \times n$ blocks as

$$
Z_{k}=\left[\begin{array}{cc}
Z_{k ; 11} & Z_{k ; 12} \\
Z_{k ; 21} & Z_{k ; 22}
\end{array}\right]
$$

we obtain the solution of PRDE at $t=(k-1) \Delta$ as

$$
X((k-1) \Delta)=Z_{k ; 21} Z_{k ; 11}^{-1}
$$

Some computational aspects are relevant for a robust implementation of this approach. Since each matrix $H_{k}$ is symplectic, it is important to employ numerical integrators which are able to guarantee this property. Note that standard methods (even the simple explicit Euler method) do not ensure that $H_{k}$ will be symplectic. Among methods able to compute symplectic solutions are the Gauss-Legendre (diagonal Padé approximants) methods which belong to the class of symplectic Runge-Kutta methods [15], [17]. MATLAB software for symplectic integration is freely available [18].

To compute the values of the solution $X(t)$ between two discretization moments $t_{0}=(k-1) \Delta$ and $t_{f}=k \Delta$, special ODE solvers as those proposed in [19], [14] can be used to integrate (4) in forward time with $X\left(t_{0}\right)=X((k-1) \Delta)$ or (5) in backward time with $X\left(t_{f}\right)=X(k \Delta)$. A distinctive feature of solvers discussed in [14] is their capability to preserve the positivity of the numerical solution of differential Riccati equations and in particular of differential Lyapunov equations.

\section{APPLICATIONS: COMPUTATION OF SYSTEM NORMS}

The proposed multi-shot techniques can be applied to solve norm computation problems for continuous-time periodic systems of the form

$$
\begin{aligned}
& \dot{x}(t)=A(t) x(t)+B(t) u(t) \\
& y(t)=C(t) x(t)+D(t) u(t)
\end{aligned}
$$

where $A(t) \in \mathbb{R}^{n \times n}, B(t) \in \mathbb{R}^{n \times m}, C(t) \in \mathbb{R}^{p \times n}$, and $D(t) \in \mathbb{R}^{p \times m}$ are periodic matrices of period $T$. In this section we assume that the periodic system $\mathcal{G}=$ $(A(t), B(t), C(t), D(t))$ is stable (i.e., $A(t)$ is stable).

System norms are important in solving many computational problems. For example, Hankel-norm based bounds appear in solving model reduction problems of continuoustime periodic systems [20], while the $\mathcal{H}_{2}$-norm and $\mathcal{H}_{\infty^{-}}$ norm play an important role in solving the periodic $\mathcal{H}_{2}$ - or $\mathcal{H}_{\infty}$-norm control synthesis problems [21], [22], [3].

All these system norms can be defined (see [21]) in terms of the input-output operator $\mathcal{G}_{o p}(\tau)$ which relates the inputs $u(t)$ and outputs $y(t)$ for zero initial conditions at $t=\tau$, i.e.,

$$
y(t)=\left[\mathcal{G}_{o p}(\tau) u\right](t), t \geq \tau
$$

Due to periodicity, the norms are defined to be independent of $\tau$ and the definitions for constant systems are recovered.

\section{A. Computation of Hankel-norm}

Analogously to the discrete-time case [23], the computation of the Hankel-norm $\left\|\mathcal{G}_{o p}\right\|_{H}$ relies on computing the positive semidefinite periodic reachability and observability Gramians $P(t)$ and $Q(t)$, respectively, defined as

$$
\begin{aligned}
& P(t)=\int_{t}^{\infty} \Phi_{A}(t, \tau) B(\tau) B^{T}(\tau) \Phi_{A}^{T}(t, \tau) \mathrm{d} \tau \\
& Q(t)=\int_{-\infty}^{t} \Phi_{A}^{T}(\tau, t) C^{T}(\tau) C(\tau) \Phi_{A}(\tau, t) \mathrm{d} \tau
\end{aligned}
$$

It is well-know that the periodic Gramians satisfy the PDLEs

$$
\begin{gathered}
\dot{P}(t)=A(t) P(t)+P(t) A^{T}(t)+B(t) B^{T}(t) \\
-\dot{Q}(t)=A^{T}(t) Q(t)+Q(t) A(t)+C^{T}(t) C(t)
\end{gathered}
$$

The Hankel-norm of the system is defined from

$$
\left\|\mathcal{G}_{\text {op }}\right\|_{H}^{2}=\max _{\tau \in[0, T]} \lambda_{\max }(P(\tau) Q(\tau))
$$

When using the multi-shot approach to solve the PDLEs (20) and (21), we have the values of $P(t)$ and $Q(t)$ in the discrete time grid points $0<\Delta<2 \Delta<\ldots<$ $(N-1) \Delta$. For practical Hankel-norm determination, we can choose a sufficiently dense time grid and compute the maximum eigenvalues only in the grid points. Furthermore, interpolation can be employed to determine the norm to higher accuracy. 


\section{B. Computation of $\mathcal{H}_{2}$-norm}

In this subsection we assume $D(t)=0$. For the computation of the $\mathcal{H}_{2}$-norm of the system (19) we mention only two recently proposed methods. The approximation-based method of [24], [25] relies on finite truncation of infinitedimensional structured matrices. For this method the choice of truncation order guaranteeing a given accuracy of the norm is the most critical aspect. Closed-form formulas to compute the $\mathcal{H}_{2}$-norm have been proposed in [26], [27]. The formula in [27] involves the evaluation of a double matrix integral by integrating a large system of ODEs which includes the computation of the transition matrices corresponding to the stable direct and unstable adjoint system dynamics. To circumvent unstable integrations, the inversion of the direct transition matrix at each time moment is necessary. Additionally, a complex matrix integral must be evaluated via complex residuals, which involves potentially sensitive computations in the case of multiple characteristic multipliers.

The straightforward method which we propose relies on the evaluation of the trace formula [3]

$$
\left\|\mathcal{G}_{o p}\right\|_{2}^{2}=\operatorname{trace}\left[\frac{1}{T} \int_{0}^{T} C(\tau) P(\tau) C^{T}(\tau) \mathrm{d} \tau\right]
$$

where $P(t)$ is the unique $T$-periodic solution of the PDLE (20). Let $\Delta=T / N$ be an appropriate discretization interval and let denote $t_{k}:=(k-1) \Delta$ and $P_{k}=P\left(t_{k}\right)$ for $k=1, \ldots, N$ the values of the solution determined by the proposed multi-shot method (to be always preferred over the periodic generator method). Then, we can compute the squared norm $\left\|\mathcal{G}_{o p}\right\|_{2}^{2}=\mu(T) / T$ by integrating (20) with initial condition $P(0)=P_{1}$ jointly with the equation

$$
\dot{\mu}(t)=\operatorname{trace}\left[C(t) P(t) C^{T}(t)\right], \mu(0)=0
$$

over the interval $[0, T]$. This computation involves (provided symmetry is exploited) the integration of $n(n+1) / 2+1$ differential equations. If the accuracy requirements are not too high, the numerical integration of (20) can be completely avoided by employing interpolation formulas to evaluate the integral in (22) based on the available $N$ discrete values trace $\left[C\left(t_{k}\right) P\left(t_{k}\right) C^{T}\left(t_{k}\right)\right], k=1, \ldots, N$.

If parallelization of computations is possible, the squared norm can be evaluated as $\left\|\mathcal{G}_{o p}\right\|_{2}^{2}=\frac{1}{T} \sum_{i=1}^{N} \mu_{k}(k \Delta)$, where each $\mu_{k}(t)$ for $t \in\left[t_{k}, t_{k+1}\right]$ satisfies

$$
\dot{\mu}_{k}(t)=\operatorname{trace}\left[C(t) P(t) C^{T}(t)\right], \mu_{k}((k-1) \Delta)=0
$$

which is integrated jointly with an equation of the form (20) with initial condition $P\left(t_{k}\right)=P_{k}$. Thus all $N$ terms of the sum can be evaluated in parallel.

\section{Computation of $\mathcal{H}_{\infty}$-norm}

The approximate computation of $\mathcal{H}_{\infty}$-norm of linear continuous-time periodic systems has been addressed in frequency domain in [24] relying on handling finite truncations of infinite-dimensional matrices. In this paper we propose a time-domain approach to the $\mathcal{H}_{\infty}$-norm computation by exploiting the characterization of the condition $\left\|\mathcal{G}_{\text {op }}\right\|_{\infty}<\gamma$ for a given $\gamma$ in terms of the characteristic multipliers of the associated Hamiltonian matrix

$$
H_{\gamma}(t)=\left[\begin{array}{cc}
\widehat{A}(t) & -\widehat{R}(t) \\
-\widehat{Q}(t) & -\widehat{A}^{T}(t)
\end{array}\right]
$$

where

$$
\begin{aligned}
& \widehat{A}(t)=A(t)+B(t)\left(\gamma^{2} I-D^{T}(t) D(t)\right)^{-1} D^{T}(t) C(t) \\
& \widehat{R}(t)=-B(t)\left(\gamma^{2} I-D^{T}(t) D(t)\right)^{-1} B^{T}(t) \\
& \widehat{Q}(t)=C^{T}(t)\left(I-D(t) D^{T}(t) \gamma^{-2}\right)^{-1} C(t)
\end{aligned}
$$

According to [21], for a stable system (19), the condition $\left\|\mathcal{G}_{o p}\right\|_{\infty}<\gamma$ is equivalent to the fact that the associated monodromy matrix $\Phi_{H_{\gamma}}(T, 0)$ does not have eigenvalues on the unit circle. This characterization can be used to compute the $\mathcal{H}_{\infty}$-norm using a standard bisection algorithm:

1. Select an upper bound $\gamma_{u b}$ and a lower bound $\gamma_{l b}$ such that $\gamma_{l b} \leq\left\|\mathcal{G}_{o p}\right\|_{\infty} \leq \gamma_{u b}$ and set $\gamma_{L}=\gamma_{l b}, \gamma_{U}=\gamma_{u b}$.

2. If $\left(\gamma_{U}-\gamma_{L}\right) / \gamma_{L} \leq$ tol (a given tolerance), then set $\left\|\mathcal{G}_{o p}\right\|_{\infty} \approx\left(\gamma_{U}+\gamma_{L}\right) / 2$, stop; otherwise go to next step.

3. Set $\gamma=\left(\gamma_{U}+\gamma_{L}\right) / 2$ and test $\left\|\mathcal{G}_{o p}\right\|_{\infty}<\gamma$ by computing the characteristic multipliers of the associated $H_{\gamma}(t)$.

4. If $H_{\gamma}(t)$ has characteristic multipliers on the unit circle, set $\gamma_{L}=\gamma$; otherwise, set $\gamma_{U}=\gamma$; go to Step 2 .

After $q$ iterations, we have $\gamma_{U}-\gamma_{L}=2^{-q}\left(\gamma_{u b}-\right.$ $\left.\gamma_{l b}\right)$. Thus, to increase the efficiency of computations it is important to have good initial approximations for the bounds. It is possible to start with a lower bound $\gamma_{l b}>$ $\max _{t \in[0, T]} \bar{\sigma}(D(t))$, where $\bar{\sigma}(\cdot)$ denotes the maximum singular value, and increase $\gamma_{l b}$ until no characteristic multipliers lie on the unit circle. The corresponding value can be taken as $\gamma_{u b}$. Alternatively, the lower bound can be taken such that $\gamma_{l b}>\max \left\{\max _{t \in[0, T]} \bar{\sigma}(D(t)),\left\|\mathcal{G}_{o p}\right\|_{H}\right\}$, while for the upper bound the value $\gamma_{u b}=\max _{t \in[0, T]} \bar{\sigma}(D(t))+2\left\|\mathcal{G}_{o p}\right\|_{H}$ can be used.

In the above algorithm we do not need to solve any periodic Riccati equation, but only to compute the eigenvalues of the monodromy matrix $\Phi_{H_{\gamma}}(T, 0)$ and check for values on the unit circle. Similarly to the case when solving a PDRE, we choose an appropriate discretization step $\Delta=T / N$, and compute $\Phi_{H_{\gamma}}(k \Delta,(k-1) \Delta)$ for $k=1, \ldots, N$ by numerical integration to obtain $\Phi_{H_{\gamma}}(T, 0)$ in a product form as in (17). As before, for numerical integrations the special symplectic integration methods are appropriate [18]. For the computation of the eigenvalues of $\Phi_{H_{\gamma}}(T, 0)$ the PRSF based algorithm can be used [8], [9].

\section{NUMERICAL EXAMPLE}

To illustrate the capabilities of the multi-shot approach, we consider the numerical solution of the PLDE (1), where we consider

$$
A(t)=\left[\begin{array}{cc}
0 & 1 \\
-10 \cos (t)-1 & -24-10 \sin (t)
\end{array}\right]
$$

and we choose $Q(t)$ such that the exact solution of (1) is

$$
X(t)=\left[\begin{array}{cc}
1+\cos (t) & 0 \\
0 & 1+\sin (t)
\end{array}\right]
$$


The period of the problem is $T=2 \pi$. The characteristic multipliers of $A(t)$ are $e^{-0.046 T}$ and $e^{-23.95 T}$, thus the problem is moderately stiff and moderately ill-conditioned.

We computed the unique periodic solution $\bar{X}(t)$ of (1) using sampling periods $\Delta=T / N$, for $N=1,16,64,128,256$. The value of $N=1$ corresponds to the one-shot method. For the discretization of the continuoous-time problem and solution of the resulting discretized periodic Lyapunov equations tools available in the PERIODIC Systems Toolbox [13] have been used. In Table 1 we present accuracy results obtained using three MATLAB ODE solvers: the DormandPrince Runge-Kutta $(4,5)$ code ode 45 , the non-stiff variable order Adams-Bashforth-Moulton solver ode113 and the numerical differentiation formulas based stiff solver ode $15 \mathrm{~s}$ all with both the relative and absolute tolerances set to $10^{-8}$. The accuracy of solution is evaluated as $\left\|X\left(t_{k}\right)-\bar{X}\left(t_{k}\right)\right\|_{2}$ in each point $t_{k}=(k-1) \Delta$, for $k=1, \ldots, N$.

TABLE I

ACCURACY RESULTS FOR $\max \left\|X\left(t_{k}\right)-\bar{X}\left(t_{k}\right)\right\|_{2}$

\begin{tabular}{cccc}
$\mathrm{N}$ & ode45 & ode113 & ode15s \\
\hline 1 & $1.6 \cdot 10^{-4}$ & $1.9 \cdot 10^{-8}$ & $2.4 \cdot 10^{-7}$ \\
16 & $1.4 \cdot 10^{-6}$ & $8.3 \cdot 10^{-9}$ & $2.1 \cdot 10^{-8}$ \\
64 & $1.1 \cdot 10^{-7}$ & $5.6 \cdot 10^{-9}$ & $5.4 \cdot 10^{-8}$ \\
128 & $9.0 \cdot 10^{-9}$ & $9.1 \cdot 10^{-9}$ & $1.1 \cdot 10^{-7}$ \\
256 & $1.1 \cdot 10^{-9}$ & $8.3 \cdot 10^{-9}$ & $8.9 \cdot 10^{-8}$
\end{tabular}

While the accuracy of the multi-shot method is always better than that of the one-shot approach, the accuracy gains obtained for the Runge-Kutta method are remarkable. Notice that the more accurate multistep methods implemented in ode113 and ode15s achieve their limiting accuracy already for $N=64$ and $N=16$, respectively.

\section{CONCLUSIONS}

We proposed several multi-shot type algorithms for solving various periodic matrix differential equations. These methods compute the periodic solutions in an arbitrary number of equidistant time instants within one period, by employing suitable discretizations of the continuous-time problems. In contrast to traditionally used one-shot periodic generator methods, the multi-shot methods have the advantage to be able to successfully tackle problems with large periods and/or unstable dynamics. We presented straightforward applications of the developed techniques in computing various periodic system norms.

All computational techniques involves as preprocessing step and sometimes also as postprocessing step the numerical integration of specific matrix differential equations. This part of computations is usually the most computer intensive processing and we assumed tacitly that existing standard or symplectic techniques can be used to perform them. It appears that in all cases these computations are "embarrassingly" parallelizable, which makes the multi-shot approach very appealing on parallel machines.

\section{REFERENCES}

[1] R. W. Brocket, Finite Dimensional Linear Systems. John Wiley, New York, 1970.
[2] G. De Nicolao and S. Strada, "On the use of reachability gramians for the stabilization of linear periodic systems," Automatica, vol. 33, pp. 729-732, 1997.

[3] P. Colaneri, "Periodic control systems: theoretical aspects," Proc. of IFAC Workshop on Periodic Systems, Yokohama, Japan, 2005.

[4] S. Bittanti, "The periodic Riccati equation," in The Riccati Equation, S. Bittanti, A. J. Laub, and J. C. Willems (Eds.), Springer-Verlag, Berlin, 1991, pp. 127-162.

[5] J. W. Choi and H. C. L. J. J.Zhu, "Decoupling and tracking control using eigenstructure assignment for linear time-varying systems," Int. J. Control, vol. 74, pp. 453-464, 2001.

[6] P. Montagnier and R. J. Spiteri, "A Gramian-based controller for linear periodic systems," IEEE Trans. Automat. Control, vol. 49, pp. 13801385,2004

[7] S. Bittanti, P. Colaneri, and G. Guardabasi, "Analysis of the periodic Lyapunov and Riccati equations via canonical decomposition," SIAM J. Control Optim., vol. 24, pp. 1138-1149, 1986.

[8] A. W. Bojanczyk, G. Golub, and P. Van Dooren, "The periodic Schur decomposition. Algorithms and applications," Proceedings SPIE Conference, F. T. Luk, Ed., vol. 1770, July 1992, pp. 31-42.

[9] J. J. Hench and A. J. Laub, "Numerical solution of the discrete-time periodic Riccati equation," IEEE Trans. Automat. Control, vol. 39, pp. 1197-1210, 1994.

[10] A. Varga, "Periodic Lyapunov equations: some applications and new algorithms," Int. J. Control, vol. 67, pp. 69-87, 1997.

[11] R. Byers and N. Rhee, "Cyclic Schur and Hessenberg-Schur numerical methods for solving periodic Lyapunov and Sylvester equations," Dept. of Mathematics, Univ. of Missouri at Kansas City, Tech. Rep., June 1995.

[12] P. Bolzern and P. Colaneri, "The periodic Lyapunov equation," SIAM J. Matrix Anal. Appl., vol. 9, pp. 499-512, 1988.

[13] A. Varga, "A Periodic Systems Toolbox for Matlab," Proc. of IFAC 2005 World Congress, Prague, Czech Republic, 2005.

[14] L. Dieci and T. Eirola, "Positive definiteness in the numerical solution of Riccati differential equations," Numerische Methematik, vol. 67, pp. 3003-313, 1994.

[15] J. J. Hench, C. S. Kenney, and A. J. Laub, "Methods for the numerical integration of Hamiltonian systems," Circuits, Systems and Signal Processing, vol. 13, pp. 695-732, 1994.

[16] J. J. Hench, 1995, (private communication).

[17] E. Hairer, C. Lubich, and G. Warnner, Geometric Numerical Integration: Structure-Preserving Algorithms for Ordinary Differential Equations. Springer-Verlag, Berlin, 2002.

[18] E. Hairer and M. Hairer, "GniCodes - Matlab programs for geometric numerical integration," in Frontiers in Numerical Analysis Durham 2002, J. Blowey et al. (Eds.), Springer, Berlin, 2003, pp. 199-240.

[19] L. Dieci, "Numerical integration of the differential Riccati equation and some related issues," SIAM J. Numer. Anal., vol. 29, pp. 781$815,1992$.

[20] H. Sandberg and A. Rantzer, "Balanced truncation of linear timevarying systems," IEEE Trans. Automat. Control, vol. 49, pp. 217-229, 2004.

[21] P. Colaneri, "Continuous-time periodic systems in $H_{2}$ and $H_{\infty}$ : Part I: Theoretical aspects," Kybernetica, vol. 36, pp. 211-242, 2000.

[22] - "Continuous-time periodic systems in $H_{2}$ and $H_{\infty}$ : Part II: State feedback problems," Kybernetica, vol. 36, pp. 329-350, 2000.

[23] S. Bittanti and P. Colaneri, "Analysis of discrete-time linear periodic systems," in Digital Control and Signal Processing Systems and Techniques, ser. Control and Dynamics Systems, C. T. Leondes (Ed.), Academic Press, 1996, vol. 78, pp. 313-339.

[24] J. Zhou and T. Hagiwara, " $H_{2}$ and $H_{\infty}$ norm computations of linear continuous-time periodic systems via the skew analysis of frequency response operators," Automatica, vol. 38, pp. 1381-1387, 2002.

[25] J. Zhou and T. Hagiwara, "Finite-dimensional models in avaluating the $\mathrm{H}_{2}$ norm of continuous-time periodic systems," Proc. of IFAC 2005 World Congress, Prague, Czech Republic, 2005.

[26] B. P. Lampe and E. N. Rosenwasser, "Statistical analysis and $\mathrm{H}_{2}$ norm of finite dimensional linear time-periodic systems," Prepr. of IFAC Workshop on Periodic Control Systems, Como, Italy, 2001, pp. 9-14.

[27] - , " $H_{2}$-norm computation for stable linear continuous-time periodic systems," Archives of Control Sciences, vol. 14, pp. 147-160, 2004. 